Direct Comparison of the X-Ray Emission and Absorption of Cerium Oxide

J. G. Tobin, S. W. Yu, B. W. Chung, G. D. Waddill, J. D. Denlinger

December 6, 2010

Journal of Vacuum Science and Technology A 
This document was prepared as an account of work sponsored by an agency of the United States government. Neither the United States government nor Lawrence Livermore National Security, LLC, nor any of their employees makes any warranty, expressed or implied, or assumes any legal liability or responsibility for the accuracy, completeness, or usefulness of any information, apparatus, product, or process disclosed, or represents that its use would not infringe privately owned rights. Reference herein to any specific commercial product, process, or service by trade name, trademark, manufacturer, or otherwise does not necessarily constitute or imply its endorsement, recommendation, or favoring by the United States government or Lawrence Livermore National Security, LLC. The views and opinions of authors expressed herein do not necessarily state or reflect those of the United States government or Lawrence Livermore National Security, LLC, and shall not be used for advertising or product endorsement purposes. 


\title{
Direct Comparison of the X-ray Emission and Absorption of Cerium Oxide ${ }^{\mathrm{a}}$
}

\author{
J.G. Tobin ${ }^{1, b, c}$, S.W. Yu ${ }^{1}$, B.W. Chung ${ }^{1}$, G.D. Waddill ${ }^{2}$, and J. D. Denlinger ${ }^{3}$ \\ 1. Lawrence Livermore National Laboratory, Livermore, CA \\ 2. Missouri University of Science and Technology, Rolla, MO \\ 3. Advanced Light Source, Lawrence Berkeley Laboratory, Berkeley, CA
}

a)This article is based on material presented at the $57^{\text {th }}$ Symposium of the American Vacuum Society: Abstract AC+TF 185, AC+TF-MoA6.

b)American Vacuum Society member

c)Corresponding Author, Tobin1@LLNL.Gov

\begin{abstract}
X-ray Emission Spectroscopy (XES) and X-ray Absorption Spectroscopy (XAS), have been used to investigate the photon emission and absorption associated with the Ce $3 \mathrm{~d}_{5 / 2}$ and Ce $3 \mathrm{~d}_{3 / 2}$ core-levels in CeOxide. A comparison of the two processes and their spectra will be made.
\end{abstract}

Materials terms: Cerium, Oxygen, X-ray Absorption Spectroscopy, X-ray Emission Spectroscopy

\section{$\underline{\text { I Introduction }}$}

Recently, we reported the observation of a very strong resonant effect in the Resonant Inverse Photoelectron Spectroscopy of CeOxide. [1] It has now become apparent that there is a utility in a direct comparison of the X-ray Emission Spectroscopy (XES) and X-ray Absorption Spectroscopy (XAS) of similarly prepared CeOxide samples. That comparison is made in this Brief Report/Comment.

\section{Experimental}

The XES experiments were carried out onsite at Lawrence Livermore National Laboratory, using a spectrometer [2] with capabilities for performing both spin resolved Fano spectroscopy [3] and high energy Inverse Photoelectron Spectroscopy (IPES) or 


\section{Direct Comparison of the X-ray Emission and Absorption of Cerium Oxide ${ }^{\mathrm{a}}$}

Bremstrahlung Isochromat Spectroscopy (BIS). [4] The XES spectra were collected using a Specs electron gun for the excitation and the XES 350 grating monochromator and channel

plate system from Scienta as the photon detection. Spectra were collected in "normal mode," where the electron gun kinetic energy (KE) and the energy position of the center of the channel plate were both fixed and the energy distribution in the photon (hv) spectrum was derived from the intensities distributed across the channel plate detector in the energy dispersal direction. The polycrystalline Ce sample was oxidized by exposure to air at ambient pressures. After introduction to the ultra-high vacuum system, the oxidized sample was bombarded with Ar, to clean the topmost surface region and stabilize the surface and near surface regions. Although $\mathrm{CeO}_{2}$ would be the thermodynamically preferred composition in an oxygen rich environment, the combination of a vacuum environment and ion etching may have driven the near surface region into a $\mathrm{Ce}_{2} \mathrm{O}_{3}$ stoichiometry. [5] XES data collection occurred with the sample at or near room temperature. The base pressure of the system was $3 \times 10^{-10}$ torr, but the pressure changed depending the energy and current of the electron gun. For example, with the XES measurements at $\mathrm{KE}=3 \mathrm{KeV}$, the pressure was approximately 8 to $9 \times 10^{-10}$ torr and the excitation current to the sample was typically $0.01 \mathrm{~mA}$. More detail of the sample preparation and analysis can be found in Reference 1. The XAS experiments were performed at Beamline 8 of the Advance Light Source, as part of a larger collaboration. $[6,7]$ The ex situ sample used at the ALS was prepared in a fashion similar that that described above.

\section{Spectral Results, Discussion and Conclusions}

A schematic of the XAS and XES processes is shown in Figure 1. To a certain extent, XAS and XES are reversed events relative to each other. However, XAS involves the 


\section{Direct Comparison of the X-ray Emission and Absorption of Cerium Oxide ${ }^{\mathrm{a}}$}

absorption of an x-ray and the movement of an electron into the unoccupied states, i.e. the Conduction Bands (CB), leaving a hole in the core level. In XES, the core hole is induced by electron bombardment and a photon is ejected in the decay process, with an electron moving from the occupied states, i.e. the Valence Bands (VB), into the core hole. The decay-process can be complicated by alternate decay-routes, as illustrated in the right-most panel. While the dominant energy contribution is the binding energy of the core level, there are also small adjustments $(\Delta)$ associated with either the CB or VB. The net effect of this is to shift the XES features to lower photon energies than the corresponding XAS features. More discussion and description of the XES and XAS processes can be found in References 1 and 6.

An example of our XES data can be seen in Figure 2. Here, there are two main peaks at $\mathrm{hv}=880 \mathrm{eV}$ and $\mathrm{hv}=898 \mathrm{eV}$, corresponding to the $\mathrm{Ce} 3 \mathrm{~d}_{5 / 2}$ and $\mathrm{Ce} 3 \mathrm{~d}_{3 / 2}$, respectively. The Ce $3 \mathrm{~d}_{5 / 2}$ exhibits substructure, associated with contributions from the underlying Ce metal and near surface CeOxide. This will be discussed more below.

The XAS results are shown in Figure 3. Our results, using a Ce sample oxidized ex situ, are shown in the middle pair and upper pair of curves. A pair of reference spectra, provided by J. Denlinger, is bottom-most. The pairs correspond to data collected using total electron yield [6] and total fluorescence yield. [10] In many respects, there is not much new here. The topmost pair and bottom-most pair, particularly the total electron yield data, reproduce results reported earlier by Kaindl et al. [8], for metallic $\mathrm{Ce}$ and $\mathrm{CeO}_{2}$, respectively. The topmost spectra are also essentially identical with our XAS data from a pristine evaporated Ce film, collected on neighboring Beam-line 7 at the ALS. [6] Interestingly, the data in the topmost pair was collected after scraping in vacuum the Ce sample that had been 


\section{Direct Comparison of the X-ray Emission and Absorption of Cerium Oxide ${ }^{\mathrm{a}}$}

oxidized ex situ. Hence, surprisingly good XAS results for metallic Ce were obtained here by preparing an ex situ oxidized sample and then scraping it in vacuum. Moreover, the middle pair of spectra, from the unscraped sample and including the effects of near surface oxidation, will be representative of the results a sample preparation process very similar to that used in the XES experiments. From the above, one can conclude that the higher photon energy features in the $3 \mathrm{~d}_{5 / 2}$ manifold arise from the oxide, while the lower photon energy features correspond to the Ce metal. This assignment is confirmed when compared to the results from the reference CeOxide film, the bottom-most pair in Figure 3. Before going on to the comparison of the XES and XAS, it should be noted that the Ce $3 \mathrm{~d}_{5 / 2}$ and Ce $3 \mathrm{~d}_{3 / 2} \mathrm{XAS}$ peaks are of approximately the same intensity. This observation is approximately consistent with the assumption of statisticality, where intensity scales with the number of core states: here 6:4 for $\mathrm{Ce} 3 \mathrm{~d}_{5 / 2}: \mathrm{Ce} 3 \mathrm{~d}_{3 / 2}$.

A visual comparison of the XES and XAS results in Figures 2 and 3 confirms the assignments to Ce metal and CeOxide, as shown in Figure 2 and discussed in Reference 1. Additionally, it is clear that the XES intensities are non-statistical, probably driven by the presence of alternate decay routes as illustrated in the right-most panel in Figure 1. Lastly, the expectation that the XES will occur at slightly lower photon energies, relative to XAS, is also confirmed.

\section{Acknowledgements}

Lawrence Livermore National Laboratory is operated by Lawrence Livermore National Security, LLC, for the U.S. Department of Energy, National Nuclear Security Administration under Contract DE-AC52-07NA27344. This work was supported by the DOE Office of Science, Office of Basic Energy Science, Division of Materials Science and Engineering. 


\section{Direct Comparison of the X-ray Emission and Absorption of Cerium Oxide ${ }^{\mathrm{a}}$}

K.T. Moore prepared the ex situ sample and aided in the data collection in the ALS

experiments. The synchrotron radiation experiments were carried out at Beamline 8 at the Advanced Light Source at Lawrence Berkeley National Laboratory, Berkeley, CA, USA.

The ALS was constructed and is operated with support from Office of Basic Energy Science in the Department of Energy. We would like to thank the staff of the ALS for their support.

\section{References}

1. J.G. Tobin, S.W. Yu, B.W. Chung, G.D. Waddill, L. Duda and J. Nordgren, "Observation of Strong Resonant Behavior in the Inverse Photoelectron Spectroscopy of Ce Oxide," under submission, 2010.

2. J.G. Tobin, S.W. Yu, T. Komesu, B.W. Chung, S.A. Morton, and G.D. Waddill, Matl. Res. Soc. Symp. Proc. 986, 63 (2007).

3. S.W. Yu and J. G. Tobin, Phys. Rev. B 77, 193409 (2008); S.W. Yu and J.G. Tobin, Surface Science Letters 601, L127 (2007).

4. J.G. Tobin, S.-W. Yu, B.W. Chung, G.D. Waddill and AL Kutepov, IOP Conf. Series: Matl Sci and Eng 9, 012054 (2010); doi:10.1088/1757-899X/9/1/012054

5. J.W. Allen, J. Magn. Mat. 47/48, 168 (1985); J. Less-Com. Met. 93, 183 (1983).

6. K.T. Moore, B.W. Chung, S.A. Morton, S. Lazar, F.D. Tichelaar, H.W. Zandbergen, P. Söderlind, G. van der Laan, A.J. Schwartz, and J.G. Tobin, Phys. Rev. B 69, 193104 (2004).

7. J. Denlinger, private communication, December 2009.

8. G. Kaindl, G. Kalkowski, W.D. Brewer, E.V. Sampathkumaran, F. Holtzberg, A. Schach v. Wittenau, J. Mag. Mag. Matl. 47/48, 181 (1985).

9. C. Bonnelle, R.C. Karnatak, and J. Sugar, Phys. Rev. A 9, 1920 (1974).

10. V. Pérez-Dieste, J. N. Crain, A. Kirakosian, J. L. McChesney, E. Arenholz, A. T. Young, J. D. Denlinger, D. L. Ederer, T. A. Callcott, S. A. Lopez-Rivera, and F. J. Himpsel, Phys. Rev. B 70, 085205 (2004).

\section{$\underline{\text { Figure captions }}$}

Figure $1 \quad$ Energy level diagrams for XES and XAS. See text for details.

Figure 2 XES results for an electron excitation kinetic energy (KE) of $3 \mathrm{KeV}$ and monochromator slits of 20 micrometers for the detector. See text for details.

Figure 3 XAS results from the ALS. See text for details. 


\section{Direct Comparison of the X-ray Emission and Absorption of Cerium Oxide ${ }^{\mathrm{a}}$}

\begin{tabular}{|c|c|c|c|c|c|}
\hline XAS Ce $3 d_{5 / 2}$ & XAS Ce $3 d_{3 / 2}$ & XES Ce $3 d_{5 / 2}$ & \multicolumn{2}{|c|}{ XES Ce $3 d_{3 / 2}$} & XES Ce $3 d_{3 / 2}$ \\
\hline $\begin{aligned} h v \approx & B E\left(3 d_{5 / 2}\right) \\
& +\Delta(C B)\end{aligned}$ & $\begin{aligned} h v \approx & B E\left(3 d_{3 / 2}\right) \\
& +\Delta(C B)\end{aligned}$ & $\begin{array}{c}\text { VB Decay } \\
\begin{array}{c}\mathrm{hv} \approx \mathrm{BE}\left(3 \mathrm{~d}_{5 / 2}\right) \\
-\Delta(\mathrm{VB})\end{array}\end{array}$ & $\begin{array}{r}\text { VB D } \\
\mathrm{hv} \approx \mathrm{BE} \\
-\angle\end{array}$ & $\begin{array}{l}\text { ecay } \\
\left.3 d_{3 / 2}\right) \\
(V B)\end{array}$ & $\begin{array}{c}3 d_{5 / 2} \text { Decay } \\
h v \approx B E\left(3 d_{5 / 2}\right) \\
-\Delta(V B)\end{array}$ \\
\hline & & & CB & & \\
\hline & & & VB & & \\
\hline $\mathrm{Ce} 3 \mathrm{~d}_{5 / 2}$ & hv & $3 \mathrm{KeV} \mathrm{hv}$ & $3 \mathrm{KeV}$ & & $3 \mathrm{KeV}$ \\
\hline $\mathrm{Ce} 3 \mathrm{~d}_{3 / 2}$ & . & & & & \\
\hline
\end{tabular}

Figure 1 


\section{Direct Comparison of the X-ray Emission and Absorption of Cerium Oxide ${ }^{\mathrm{a}}$}

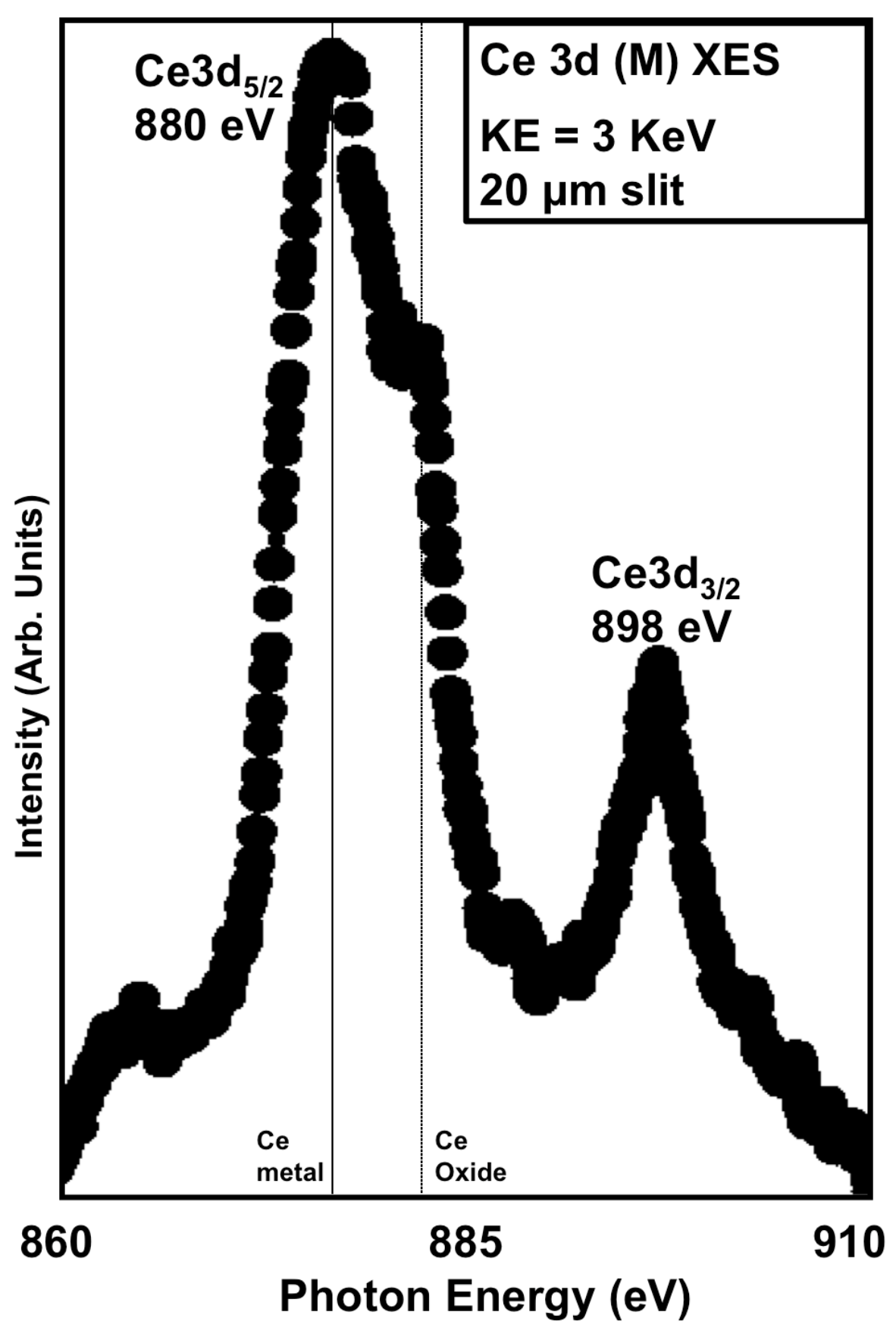

Figure 2 


\section{Direct Comparison of the X-ray Emission and Absorption of Cerium Oxide ${ }^{\mathrm{a}}$}

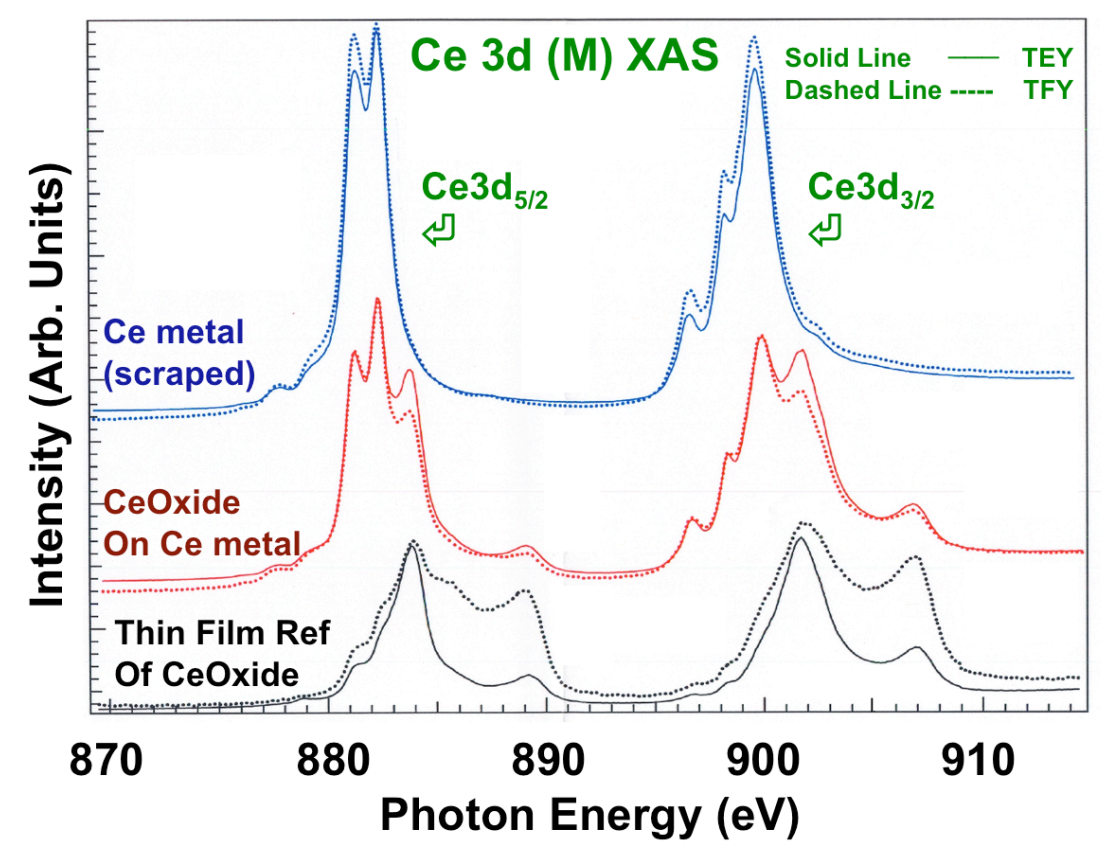

Figure 3 